HORIZON LATIN ET FRANÇAIS 



\title{
Les voyages de l'empereur Hadrien : des sources antiques à Mémoires d'Hadrien
}

\author{
Rémy Poignault
}

L'empereur Hadrien, qui a régné d'août 117 à juillet 138, donc pendant près de 21 ans, a passé presque douze ans en déplacement dans les provinces de l'Empire, et les auteurs antiques mettent l'accent sur cette originalité. Eutrope, auteur au IV ${ }^{\mathrm{e}} \mathrm{s}$. d'un abrégé de l'histoire romaine peut dire de lui : orbem Romanum circumiit : multa aedificauit (8.7.2: "Il fit tout le tour du monde romain et y édifia de nombreuses constructions", trad. J. Hellegouarc'h, Les Belles Lettres, 1999). La Vie d'Hadrien (13.5) dans l'Histoire Auguste, recueil de biographies

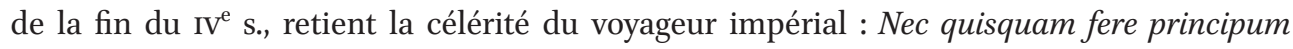
tantum terrarum tam celeriter peragrauit ("presque aucun prince ne parcourut si vite tant de pays", trad. J.-P. Callu et al., Les Belles Lettres, 2002). Fronton, contemporain d'Hadrien, souligne l'importance de ces voyages et les lie à l'activité de bâtisseur d'Hadrien comme à l'exercice du pouvoir $\left(A l s ., 5.229 ; 15^{-230.2^{1}}\right)$, mais il en parle aussi avec une certaine ironie (Hist., 11, p. 209, 1-6). Le successeur d'Hadrien, Antonin, sera, pour sa part, sédentaire (HA, Vie d'Antonin le Pieux, 7.11), et son contre-exemple pourra peut-être servir à une critique post mortem (Ael. Aristide, Rom., 33) ${ }^{2}$.

\section{HADRIEN ET SES PRÉDÉCESSEURS}

Certes ses prédécesseurs ne sont pas restés, en général, confinés en Italie pendant leur règne. Mais les voyages des prédécesseurs d'Hadrien, à l'exception, sans doute d'Auguste, font bien pâle figure en comparaison des siens ${ }^{3}$.

Hadrien assume pleinement la tradition du voyage impérial instaurée par Auguste et reprise par plusieurs de ses successeurs. Mais, prince aimant la paix, Hadrien, à quelques exceptions près, ne se met pas en route pour diriger des opérations militaires, même si la dimension militaire du voyage, en particulier par des inspections de troupes et l'édification ou la consolidation du limes est loin d'être négligeable. En outre, si le monde romain n'a peut-être pas besoin d'une réorganisation aussi importante qu'après les ravages des guerres

1 Nous citons Fronton d'après l'édition de Van den Hout, M. Cornelii Frontonis Epistulae, Leipzig, Teubner, 1988, le chiffre de la page est suivi de l'indication de la ligne.

2 Pour une étude de la position des sources littéraires par rapport au goût d'Hadrien pour le voyage, $\mathrm{cf}$. Birley 2003.

3 Sur les voyages des empereurs, voir, en particulier, Halfmann 1986 ; Chevallier 1988, 175-203 ; André 1993, 172-192. 
civiles, le voyage est un mode de gouvernement qui va de pair avec la prise de conscience de l'importance croissante du rôle des provinces. Le voyage impérial, militaire, administratif, politique, religieux, obéit aussi à des aspirations et curiosités personnelles, culturelles, voire touristiques. À mi-chemin des expéditions militaires de Trajan et de la tournée artistique d'un Néron, les voyages d'Hadrien relèvent d'une pratique délibérée et rationnelle du pouvoir, et, s'ils concernent souvent la partie grecque de l'empire, ils ne négligent pas l'Occident ; mais ils sont aussi le fait d'un être curieux de tous les savoirs, y compris le secret caché des choses.

\section{LES ITINÉRAIRES D'HADRIEN}

Il ne s'agit pas pour nous ici de déterminer l'itinéraire précis des voyages d'Hadrien. Il nous suffira de constater qu'il s'est déplacé à peu près partout dans le monde gréco-romain 4 . Avant son accession, nous savons qu'il s'est rendu en Espagne, à Italica, d'où sa famille était originaire, qu'il alla à Athènes, au moins en 111-112, année où il fut archonte de la ville, honneur tout à fait singulier pour un non Grec. Il exerça, en outre, différentes fonctions propres à lélite sénatoriale au service de l'État en Pannonie, en Mésie inférieure et en Germanie supérieure ; il participa aux guerres daciques et accompagna Trajan dans sa guerre contre les Parthes.

Pendant son règne, ses voyages sont très nombreux. Il n'est pas toujours aisé de restituer l'itinéraire d'Hadrien. Pour ce faire, on s'appuie sur les sources littéraires (comme les parties apparemment chronologiques de la Vie d'Hadrien ${ }^{5}$ ), des inscriptions, des papyri, des ostraca, des monnaies ; mais ces documents ne sont pas toujours datés et l'on ne sait pas toujours s'il faut les rattacher à tel ou tel voyage quand Hadrien s'est rendu à plusieurs reprises dans la même zone ; en outre toutes les inscriptions locales remerciant Hadrien pour ses bienfaits n'impliquent pas forcément la venue du prince en personne.

Dans l'écrit à caractère autobiographique que lui prête Marguerite Yourcenar, Mémoires d'Hadrien (ici abrégé Had.), publié en 1951, l'auteur retrace ces voyages en suivant les travaux scientifiques modernes, mais elle se tire très habilement des difficultés chronologiques en jouant des incertitudes de la mémoire :

"Les dates se mélangent : ma mémoire se compose une seule fresque où s'entassent les incidents et les voyages de plusieurs saisons. La barque luxueusement aménagée du marchand Érastos d'Éphèse tourna sa proue vers l'Orient, puis vers le sud, enfin vers cette Italie qui devenait pour moi l'Occident. Rhodes fut touchée deux fois ; Délos, aveuglante de blancheur, fut visitée d'abord par un matin d'avril et plus tard sous la pleine lune du solstice; le mauvais temps sur la côte d'Épire me permit de prolonger une visite à Dodone". (Yourcenar, Had., $412^{6}$ )

On trouvera des renseignements précis dans Halfmann 1986, 188-210 ; Chevallier 1988, 190-194 ; Birley 1997 ; Chevallier \& Poignault 1998, 47-54 ; Birley 2003, 429-432 ; Roman 2008, 168-194.

5 Voir, en particulier, le long passage de la Vie d'Hadrien (11.2-14.7), qui apparaît comme suivant l'empereur dans ses déplacements.

6 Nous citons Mémoires d'Hadrien dans CEuvres romanesques, Paris, Gallimard, coll. "Bibliothèque de la Pléiade", 2005 . 
Quand Hadrien apprend la mort de Trajan, en août 117, il se trouve à Antioche en Syrie, en qualité de legatus Augusti pro praetore et de commandant en chef de l'armée parthique. Mais il ne fait pas son entrée dans l'Vrbs avant juillet 118.

Après deux ans environ passés à Rome, le prince entame un voyage de près de cinq ans.

La première grande tournée impériale (peu après le 21 avril 121-125)

Par la Gaule, Hadrien gagne les deux provinces de Germanie ${ }^{7}$. On le retrouve ensuite en Bretagne en 122, où il met au point une frontière fortifiée dans le Nord du pays, qui marque la fin de l'ère d'expansion territoriale des Romains : c'est le fameux "mur d'Hadrien". Puis il passe par la Gaule, va en Espagne. Au début de 123 la menace que font peser les Parthes le contraint à se rendre dans la partie orientale de l'empire, en Syrie. Il voyage dans l'Est de l'empire. De Rhodes, par les Cyclades, il se dirige vers Athènes et va passer un an en Grèce.

Il ne rentre pas directement par Brindes en Italie, ce qui serait l'itinéraire le plus logique, mais il fait voile vers la Sicile, où il fait l'ascension de l'Etna pour admirer depuis son sommet le lever du soleil.

Mi-août-début septembre 125, l'empereur est à Tibur, d'où il envoie une lettre à l'Amphictyonie delphique. Les Fasti Ostienses nous apprennent que le 3 mars 127 il quitte Rome pour un voyage en Italie du Nord, dans la vallée du Pô, ce qui montre que son attention ne se porte pas seulement vers les provinces.

Et en 128 il entame, par l'Afrique, une seconde grande tournée impériale.

\section{Seconde grande tournée impériale (été 128-134)}

Hadrien est le premier empereur à se rendre en Afrique ; il y a beaucoup de traces de ses bienfaits en Afrique. Ce qu'on retient surtout de ce voyage, c'est la tournée d'inspection militaire au quartier général de Lambèse, en Numidie, du $1^{\mathrm{er}}$ au 12-13 juillet 128 ; une inscription a préservé, en effet, une partie des allocutions prononcées par Hadrien à cette occasion.

Peut-être poussa-t-il jusqu'en Maurétanie. Il rentre en Italie, où il ne reste pas plus de deux mois, pour surveiller, en particulier, le grand programme de constructions qu'il a lancé.

Son premier but est la Grèce, où on le retrouve en septembre 128. En mars 129 au plus tard il part pour l'Asie Mineure. Sur la route de la Judée, il passe en Arabie où une porte monumentale est élevée en son honneur à Gerasa.

Il part ensuite pour l'Égypte.

Hadrien arrive à Alexandrie en juillet-août 130 ; il se rend à Heliopolis, où un grand papyrus magique nous informe qu'il a assisté à une démonstration de sortilèges accomplie par le prêtre Pachratès ${ }^{8}$. 
Hadrien poursuit, malgré son deuil à la suite de la mort d'Antinoüs, sa croisière vers le Sud. Il est près de Thèbes les 18-21 novembre 130, où il rend visite au colosse de Memnon.

Il quitte l'Égypte sans doute au printemps 131 après y avoir passé sept ou huit mois.

Il arrive ensuite en Grèce, où il fait ainsi sa troisième visite en tant qu'empereur. Nous savons grâce à une lettre officielle que l'empereur est à Rome le 5 mai 134.

À partir de mai 134, Hadrien ne quittera plus l'Italie, occupé qu'il est à régler des questions depuis Rome, dont la moindre n'est pas celle de sa succession.

\section{COMMENT HADRIEN VOYAGE-T-IL ?}

Les sources littéraires et l'épigraphie contiennent quelques données qui nous permettent d'imaginer un peu les conditions dans lesquelles voyageait l'empereur.

Dion Cassius (69.10.1) ${ }^{9}$ nous apprend qu'il parcourut les villes de l'Empire sans l'apparat impérial, dont il n'usait qu’à Rome. L'Epitome de Caesaribus, qui date de la fin du Iv s. est, en fait, le plus riche en informations :

Immensi laboris, quippe qui prouincias omnes passibus circumierit, agmen comitantium praeuertens, cum oppida uniuersa restitueret, augeret ordinibus. Namque ad specimen legionum militarium fabros, perpendiculatores, architectos genusque cunctum exstruendorum moenium seu decorandorum in cohortes centuriauerat.

"D’une activité sans bornes, il parcourut à pied toutes les provinces en précédant la troupe de ses accompagnateurs ; il restaurait toutes les cités et en augmentait l'importance grâce aux corporations. En effet, sur le modèle des légions de l'armée, il avait organisé en centuries et en cohortes les ouvriers, métreurs, architectes et tous les métiers compétents pour la construction et la décoration des édifices". (Ps. Aur.-Vict., Caes., 14.4-5 ${ }^{10}$ )

Il faut peut-être faire la part de l'exagération, mais si Hadrien se déplace avec une telle compagnie, cela n'est nullement contradictoire avec la simplicité relevée par Dion Cassius : il n'a pas d'équipage et il précède ses accompagnateurs, pour, sans doute, évaluer les besoins sur place ; nous touchons là une finalité essentielle des voyages : la restauration et l'embellissement architectural des villes. Ces hommes de l'art organisés selon le modèle militaire, ou - selon une autre interprétation - ces soldats transformés en constructeurs, sont à lire en parallèle avec la troupe qui accompagnait Néron au cours de son voyage en Grèce ; ils en constituent comme l'antithèse. En effet, Néron avait organisé sa suite comme une armée, - avec les Augustiani ainsi que des musiciens, des acteurs ${ }^{11}$ - de façon à assurer ses succès artistiques, tandis qu'Hadrien voyage pour œuvrer au bien-être matériel de ses sujets et à la beauté de l'empire. à l'appareil impérial, car jamais il ne l'employa hors de Rome". 
On peut se faire une idée des compagnons de voyage de l'empereur d'après certains documents et en se livrant à des suppositions de bon sens : l'empereur devait être accompagné d'un détachement de prétoriens pour assurer sa protection, de membres de la chancellerie impériale, du Consilium principis, car les voyages n'empêchent pas l'empereur de mener à bien les affaires de l'empire, et Hadrien, bien qu'excentré, reste en contact avec l'ensemble du monde romain ; il a, d'ailleurs, amélioré l'organisation du cursus publicus, système de poste publique servant à la circulation des messagers impériaux et des serviteurs de l'État ; de même, il a réorganisé les rouages de l'administration dans le sens d'une plus grande centralisation. Hadrien doit être accompagné aussi d'un certain nombre d'esclaves et d'affranchis de la maison impériale. Nous savons aussi que l'impératrice, Antinoüs ou Lucius Ceionius Commodus ont fait partie de certains voyages au moins.

Se pose aussi la question de l'hébergement de l'empereur au cours de ses déplacements. Il a dû connaître des conditions très différentes selon qu'il était aux avant-postes militaires ou dans quelque brillante capitale provinciale. Les palais des gouverneurs de province ont dû le recevoir assez souvent ; mais il a pu loger aussi bien chez des amis ${ }^{12}$ : à Athènes, il a sans doute été l'hôte du richissime Claudius Atticus (père du fameux sophiste Hérode Atticus) devenu sénateur romain. Mais ce ne sont là que des suppositions, même si elles sont fondées. Mémoires d'Hadrien adopte cette hypothèse pour le séjour athénien qui suivit la rencontre avec Antinoüs :

"Ce bel hiver fut riche en fréquentations amicales : l'opulent Atticus, dont la banque finançait mes travaux édilitaires, non sans d'ailleurs en tirer profit, m’invita dans ses jardins de Képhissia, où il vivait entouré d'une cour d'improvisateurs et d'écrivains en vogue ; son fils, le jeune Hérode, était un causeur à la fois entraînant et subtil ; il devint le commensal indispensable de mes soupers d'Athènes". (Yourcenar, Had., p. 409-410)

$\mathrm{Au}$ cours d'un autre voyage, après la disparition du favori, il réside selon Mémoires d'Hadrien (p. 453), cette fois, chez son ami Arrien ${ }^{13}$, dans une atmosphère totalement différente, puisqu'il évoque la quiétude du couple formé par Arrien et son épouse et qu'il signale qu'Arrien, comme lui, a été initié aux mystères d'Éleusis : l'atmosphère est religieuse, littéraire (il est question immédiatement après de la bibliothèque instaurée par Hadrien à Athènes) : cela correspond à la période de Disciplina Augusta, où pas à pas Hadrien essaie de surmonter l'épreuve du malheur. On voit bien la mise en œuvre littéraire de ces données ou hypothèses : pour Marguerite Yourcenar, le choix de l'hôte n'est pas innocent, Atticus correspondant à l'euphorie de Saeculum aureum et Arrien à la reconquête d'une forme de sagesse.

Des documents épigraphiques nous montrent que de riches notables ont contribué à son accueil pour ce qui est de questions d'intendance. Nous n'en retiendrons qu'un exemple.

12 Birley 1997, 217.

13 Historiquement, il n'est pas sûr que l'empereur ait pu être alors l'hôte d'Arrien ; la chronologie de ce grand commis de l'État manque de netteté, mais il a dû être consul suffect en 129 ou 130, puis légat de Cappadoce au début de 131 ou même en 130 (Bosworth 1993, 230), et il ne s'installa définitivement à Athènes qu'en 137 . 


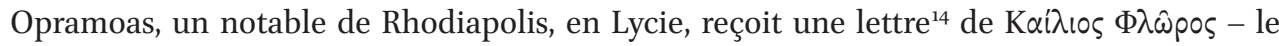
procurateur Caelius Florus - qui tient à s'assurer que tout le nécessaire sera fait pour que le séjour d'Hadrien soit le plus agréable possible : il a déjà contacté officiellement les instances de la cité, mais il tient à ce qu'Opramoas veille personnellement à ce que toutes les dispositions soient prises ; le procurateur rappelle les excellentes relations qu'il entretient avec son correspondant. On voit ici comment le pouvoir romain en appelle aux élites locales pour la réussite du voyage.

Marguerite Yourcenar utilise ce personnage en faisant de lui un utile intermédiaire dans ses négociations orientales : ce "marchand" envoie des "rapports secrets" sur la situation en Orient à Hadrien dès avant son accession (Had., p. 349) ; plus tard, il facilite les négociations parce qu'il "avait l'oreille des satrapes" (Had., p. 359). Et Hadrien nous donne une idée de la manière dont il pouvait être reçu dans ses déplacements : "Je m’arrêtai une heure en Lycie pour décider le marchand Opramoas, qui avait déjà prouvé ses qualités de négociateur, à m'accompagner en territoire parthe. Le manque de temps l'empêcha de déployer son luxe habituel. Cet homme amolli par l'opulence n'en était pas moins un admirable compagnon de route, accoutumé à tous les hasards du désert" (Had., p. 395).

On dispose aussi de quelques renseignements sur les moyens de transport. On sait ainsi, par une lettre de recommandation d'Hadrien ${ }^{15}$ adressée aux Éphésiens pour soutenir la candidature d'un certain Érastos au Sénat local en 129, que cet armateur a accompagné par mer l'empereur à deux reprises, pour aller d'Éphèse à Rhodes, d'une part, et d'Éleusis à Éphèse, d'autre part. On a longtemps cru qu'Érastos était un ami d'Hadrien et que celui-ci avait voyagé sur son bateau. En réalité, Hadrien, dans un autre document ${ }^{16}$, soutient dans les mêmes termes un autre nauclère, Philokyrios, qui a rendu pareils services sur les mêmes itinéraires : nous sommes, en fait, devant des armateurs qui ont mis à la disposition des autorités romaines leur flotte pour assurer le transport des personnes et des biens lors de ces déplacements de l'empereur et de son entourage ${ }^{17}$.

Dans Mémoires d'Hadrien, Érastos est un marchand qui met à la disposition de l'empereur une "barque luxueusement aménagée" (Had., p. 412, 408) pour se déplacer en Méditerranée. Ce qui ressort surtout de l'ouvrage, c'est la volonté délibérée d'Hadrien d'être ouvert à toutes les sollicitations et de goûter l'extrême diversité des moyens de locomotion, comme des gîtes et des usages locaux :

Sur vingt ans de pouvoir, j'en ai passé douze sans domicile fixe. J'occupais à tour de rôle les palais des marchands d'Asie, les sages maisons grecques, les belles villas munies de bains et de calorifères des résidents romains de la Gaule, les huttes ou les fermes. La tente légère, l'architecture de toile et de cordes, était encore la préférée. Les navires n'étaient pas moins

IGRR, III, 739 ; Smallwood 1966, $\mathrm{n}^{\circ} 497$.

Smallwood 1966, $\mathrm{n}^{\circ} 72 \mathrm{a} ;$ Börker \& Merkelbach 1980, $\mathrm{n}^{\circ} 1487$.

Börker \& Merkelbach 1980, $\mathrm{n}^{\circ} 1488$.

Drew-Bear \& Richard 1994. 
variés que les logis terrestres : j'eus le mien, pourvu d'un gymnase et d'une bibliothèque, mais je me défiais trop de toute fixité pour m'attacher à aucune demeure, même mouvante. La barque de plaisance d'un millionnaire syrien, les vaisseaux de haut bord de la flotte, ou le caïque d'un pêcheur grec convenaient tout aussi bien. Le seul luxe était la vitesse et tout ce qui la favorise, les meilleurs chevaux, les voitures les mieux suspendues, les bagages les moins encombrants, les vêtements et les accessoires les mieux appropriés au climat. Mais la grande ressource était avant tout létat parfait du corps : une marche forcée de vingt lieues n'était rien, une nuit sans sommeil n'était considérée que comme une invitation à penser. Peu d'hommes aiment longtemps le voyage, ce bris perpétuel de toutes les habitudes, cette secousse sans cesse donnée à tous les préjugés. Mais je travaillais à n’avoir nul préjugé et peu d'habitudes. (Yourcenar, Had., p. 380-381)

Cet art de voyager n'est pas sans faire penser à Montaigne, qui nous dit : "le voyager me semble un exercice profitable. L'âme y a une continuelle exercitation à remarquer les choses incogneuës et nouvelles ; et je ne sçache point meilleure escolle, comme j'ay dict souvent, à former la vie que de luy proposer incessamment la diversité de tant d'autres vies, fantasies et usances, et luy faire gouster une si perpetuelle varieté de formes de nostre nature" ${ }^{m 18}$.

\section{FONCTION DES VOYAGES}

Les finalités de ces voyages sont multiples.

Certes les raisons proprement "touristiques" ne sont pas à exclure. Hadrien, homme très cultivé, prend plaisir à découvrir sur place des lieux qui chantent dans sa mémoire, car il les a rencontrés pour la première fois dans les livres et il se trouve un peu dans une situation analogue à celui qui a fait ses "humanités" et visite à la faveur d'un voyage en Grèce des sites littéraires célèbres. La Vie d'Hadrien (17.8) souligne cette motivation en y ajoutant une curiosité d'ordre géographique : Peregrinationis ita cupidus ut omnia quae legerat de locis orbis terrarum praesens uellet addiscere ("Il avait une telle passion des voyages qu'il voulut s'informer sur place de tout ce qu'il avait lu des régions de la terre"19). Il y a chez Hadrien une curiosité "archéologique", comme celle qui anima Germanicus en 18-19 a.C. et conduisit ce prince en Grèce, en Asie, sur le site de Troie, mais aussi en Égypte, où il se renseigna semblablement sur le passé et la langue des Égyptiens (Tac., Ann., 2.53-55; 59). Hadrien se rend sur les tombes des grands hommes de l'histoire ou du mythe : Alcibiade ${ }^{20}$ (Had., p. 413-414), Épaminondas ${ }^{21}$ (Had., p. 408), Pompée ${ }^{22}$ (Had., p. 430), mais aussi Ajax et peutêtre Hector. Le voyage est ainsi une sorte de pèlerinage culturel chez cet ardent philhellène. À Péluse, il restaure le tombeau de Pompée, où il fait inscrire un vers : "Pour celui qui fut 
si prodigue de temples, quelle indigence dans son tombeau" (Anth., 9.402 ; D. C. 69.11.1) : dans Mémoires d'Hadrien (p. 430), il dit son admiration pour l'œuvre de Pompée en Orient. Hadrien se recueille aussi sur les tombes des héros de la guerre de Troie ; on se rappelle que dans la Pharsale (9.950 sq.) de Lucain, Jules César se rend sur les ruines de Troie et manque de fouler aux pieds la tombe d'Hector, qui est presque imperceptible ; pour Hadrien, cette visite est l'occasion d'évoquer la différence de perception d'Antinoüs et lui-même et de nous faire sentir la distance qui s'instaure entre eux :

"Au cours d'un voyage en Troade, nous visitâmes la plaine du Scamandre sous un ciel vert de catastrophe : l'inondation, dont j'étais venu sur place constater les ravages, changeait en îlots les tumulus des tombeaux antiques. Je trouvai quelques moments pour me recueillir sur la tombe d'Hector ; Antinoüs alla rêver sur celle de Patrocle. Je ne sus pas reconnaître dans le jeune faon qui m'accompagnait l'émule du camarade d'Achille : je tournai en dérision ces fidélités passionnées qui fleurissent surtout dans les livres ; le bel être insulté rougit jusqu'au sang. La franchise était de plus en plus la seule vertu à laquelle je m’astreignais : je m’apercevais que les disciplines héroïques dont la Grèce a entouré l'attachement d'un homme mûr pour un compagnon plus jeune ne sont souvent pour nous que simagrées hypocrites". (Yourcenar, Had., p. 424)

L'empereur va voir aussi des mirabilia, comme le colosse de Memnon, en fait la statue du pharaon Aménophis III ; une fissure dans la pierre en faisait une curiosité car elle émettait souvent un son au lever du soleil, quand la pierre se réchauffait. Des inscriptions laissées sur la jambe du colosse par la poétesse Julia Balbilla, comme le firent bien d'autres touristes antiques, rappellent la visite impériale ${ }^{23}$. Le premier jour, la statue est restée muette, mais le lendemain elle s'est fait entendre à la $1^{\text {ère }}$ heure à Sabine et à la poétesse, et, à la $2^{\mathrm{e}}$ heure, à Hadrien. L'épisode est relaté dans Mémoires d'Hadrien (Had., p. 444-445) et comme il s'est produit peu après la mort d'Antinoüs, il est empreint d'une immense mélancolie, Hadrien étant surtout sensible à la mort de la civilisation égyptienne.

Il y a aussi chez Hadrien un certain goût pour le spectacle de la nature. Marguerite Yourcenar lui prête une fascination pour les immenses paysages déserts ( $\mathrm{Had} ., 321$ sq.) en se fondant, outre ce que les textes nous apprennent de son goût des voyages, sur un objet archéologique, un vase représentant un envol d'échassiers trouvé à la Villa Adriana ("Carnets de notes de Mémoires d'Hadrien", p. 534-535). Le prince connaît, selon Marguerite Yourcenar, une véritable exaltation devant la découverte de paysages totalement nouveaux pour ce méditerranéen : le caractère grandiose de la vallée du Danube et l'absolue pureté cristalline des steppes sous la neige et la glace :

"Notre sol grec ou latin, soutenu partout par l'ossature des rochers, a l'élégance nette d'un corps mâle : la terre scythe avait l'abondance un peu lourde d'un corps de femme étendue. La plaine ne se terminait qu'au ciel. Mon émerveillement ne cessait pas en présence du miracle des fleuves : cette vaste terre vide n'était pour eux qu'une pente et qu'un lit. [...] on galopait dans un monde de pur espace et d'atomes purs. Aux choses les plus banales, les plus molles, 
le gel donnait une transparence en même temps qu'une dureté céleste". (Yourcenar, Had., p. 321-322)

Hadrien va admirer le lever du soleil depuis le sommet de l'Etna. Selon la Vie d'Hadrien (13.3), Post in Siciliam nauigauit, in qua Aetnam montem conscendit, ut solis ortum uideret arcus specie, ut dicitur, uarium. ("Ensuite, il se rendit par mer en Sicile où il fit l'ascension du mont Etna pour voir le lever du soleil aux couleurs variées, dit-on, comme celles d'un arc-en-ciel"), ce qui est mettre en avant le plaisir esthétique de contemplation de la nature. On peut voir là sans doute, outre le plaisir du spectacle de la nature, une forme de curiosité scientifique qui s'est développée à Rome depuis le $\mathrm{II}^{\mathrm{e}}$ s. a.C. avec Panétius et son disciple Posidonius au siècle suivant, et qui pousse les Romains à s'intéresser aux fleuves, aux montagnes et aux volcans. Mais les Romains ne pratiquent guère l'escalade en général et les ascensions d'Hadrien n'en sont que plus originales ${ }^{24}$. Ces dimensions sont tout à fait présentes dans Mémoires d'Hadrien, où l'empereur est accompagné de savants, mais l'épisode reçoit, en outre, une valeur symbolique, puisque Hadrien nous dit : "Ce fut l'une des cimes de ma vie. Rien n'y manqua, ni la frange dorée d'un nuage, ni les aigles, ni l'échanson d'immortalité".

L'ascension du mont Casios, où il se trouvait a davantage une valeur religieuse. La Vie d'Hadrien (14.3) la présente ainsi : Sed in monte Casio, cum uidendi solis ortus gratia nocte ascendisset, imbre orto fulmen decidens hostiam et victimarium sacrificanti adflauit. (“Cependant, tandis qu'il offrait un sacrifice sur le Mont Casios, dont il avait fait l'ascension de nuit pour voir le lever du soleil, un orage survint et la foudre, en tombant, atteignit de son souffle la victime et le victimaire"). Cet épisode est orchestré dans Mémoires d'Hadrien de manière à être mis en parallèle avec l'ascension de l'Etna et faire ressortir l'assombrissement de l'atmosphère, nous préparant à l'idée du sacrifice d'Antinoüs (Had., p. 428-429).

Ainsi le tourisme d'Hadrien peut prendre une tonalité religieuse, puisque sur le mont Casios se trouvait un sanctuaire de Zeus-Casios. Toujours est-il qu'Hadrien s'est rendu dans les sanctuaires célèbres et a été initié à des cultes à mystères, dont les plus grands sont ceux d'Éleusis. Peut-être a-t-il été initié aux mystères des Cabires à Samothrace. Si l'on en croit Jérôme, hyperbolique en la matière, Hadrien aurait été initié à presque tous les sacra de la Grèce (Vir. ill., 19) et Tertullien le présente comme omnium curiositatum explorator ("partant à la découverte de toutes les choses curieuses", Apol., 8). Hadrien restaure, en outre, de nombreux temples ou en achève d'autres, comme l'Olympieion à Athènes ; il contribue au redressement du sanctuaire d'Apollon à Delphes, reconstruit un nouveau Poseidônion à Mantinée (cf. Had., p. 408)...

Outre le soin porté à la religion traditionnelle, le voyage révèle le souci de développer dans les provinces le culte impérial ${ }^{25}$ : dans tout l'Orient, Hadrien est vénéré comme un dieu à part entière, seul ou partageant le temple d'une divinité du panthéon traditionnel. Il est nouveau Dionysos, Zeus Olympien. Dans le Panhellénion, à Athènes, sa statue trône à 
côté de celle de Zeus Panhellénios. Le développement et la réorganisation du culte impérial contribuent à asseoir la dynastie et à affermir le loyalisme des sujets : l'empereur, par sa présence dans les provinces et par le culte impérial qui s'y développe confère à cet immense empire un plus grand sentiment d'unité, et il montre à tous le prince, dont les provinciaux ne pouvaient guère voir jusque-là que l'effigie sur les monnaies ou dans la statuaire.

Même une activité qui pourrait apparaître comme un pur passe-temps, la chasse, peut revêtir une plus haute portée symbolique ${ }^{26}$. Il y a bien là un goût personnel très fort d'Hadrien. Il aime chasser le sanglier, l'ours ou le lion ; il est très lié à son cheval Borysthène, compagnon de ses chasses ; il immortalise celles-ci dans les tondi qui seront réemployés dans l'arc de Constantin ; il donne à l'une des villes qu'il fonde, en Mysie, un nom qui rappelle un exploit cynégétique - Hadrianotherae ("les Chasses d'Hadrien" - quod illic et feliciter esset uenatus et ursam occidisset aliquando ("parce qu'il avait chassé là avec succès et qu'une fois il avait tué une ourse"), nous dit la Vie d'Hadrien (20.13). Dion Cassius va dans le même sens en affirmant qu'il se cassa une clavicule et faillit se briser une jambe à la chasse (69.10.2). Mais le plaisir personnel que l'empereur pouvait prendre à cette passion cynégétique va de pair avec une fonction politico-religieuse de ces chasses. Pour Hadrianotherae, comme pour Antinoé, il s'agit pour l'empereur de ressouder le tissu social d'une région en créant un centre de développement de la culture gréco-romaine. En outre, en diffusant de lui-même l'image d'un chasseur, il exalte une forme de uirtus qui n'est plus guerrière, comme sous Trajan, mais est tout aussi éclatante.

Les voyages sont aussi l'occasion de se rendre compte personnellement des besoins des provinces et de mettre en ouvre des programmes de construction ou reconstruction. À Carthage, qui devient Hadrianopolis (Vie d'Hadrien, 20.4), il contribue à la construction d'un aqueduc ; son évergétisme prend une dimension quasi-miraculeuse, puisque, selon la Vie d'Hadrien (22.14), son arrivée dans la province coïncida avec la première pluie depuis cinq ans. Les Mémoires d'Hadrien s'en font l'écho : "Le jour de mon arrivée à Carthage, une sécheresse de cinq ans prit fin ; la foule délirant sous l'averse acclama en moi le dispensateur des bienfaits d'en haut ; les grands travaux d'Afrique ne furent ensuite qu'une manière de canaliser cette prodigalité céleste" (Had., p. 421). Les voyages sont aussi l'occasion de prendre des mesures administratives : par exemple, en Afrique, il améliore le statut juridique de certaines cités de manière à faciliter l'intégration : Bulla Regia et Zama Regia deviennent ainsi des colonies et d'autres communautés reçoivent le statut de municipe ${ }^{27}$.

Les voyages impériaux relèvent beaucoup de l'exercice direct du pouvoir. Hadrien se rend sur place pour inspecter les armées et les lignes de défense. L'empereur passe en revue les troupes, dirige des manœuvres et fait connaître son avis, comme à Lambèse ${ }^{28}$ (Had., p. 421-422) ; il veille à la discipline, instituant le culte de la Disciplina Augusta. Des 
monnaies du type Exercitus vont témoigner de l'intérêt d'Hadrien pour les armées, le prince étant d'autant plus porté à veiller au bon fonctionnement de l'instrument militaire qu'il a des intentions pacifiques. Par ses déplacements, en outre, Hadrien peut mieux se rendre compte de l'administration des provinces et il exerce aussi une surveillance sur les serviteurs de l'État : selon la Vie d'Hadrien (13.10), Et circumiens quidem prouincias procuratores et praesides pro factis supplicio adfecit, ita seuere ut accusatores per se crederetur inmittere ("Par ailleurs, en faisant le tour des provinces, il infligea à des procurateurs et à des gouverneurs des peines en rapport avec leurs actions et fit preuve d'une sévérité telle que l'on crut que c'était lui qui suscitait les accusateurs"). Hadrien est donc à l'écoute des doléances des provinciaux et soucieux de justice. Il évalue aussi les besoins des villes et des provinces, met en œuvre, finance ou suscite de grands travaux de constructions pour améliorer le bien-être des populations ou redonner plus d'éclat aux cités. Les voyages sont l'occasion de manifester sur toute l'étendue de l'empire l'évergétisme impérial. Ils sont aussi l'occasion pour le prince de choisir l'emplacement de nouvelles fondations de cités pour répondre à des besoins économiques et accentuer la romanisation et l'hellénisation.

Le voyage est donc inhérent à la conception qu'Hadrien se fait de sa fonction d'empereur. Il révèle - comme le font aussi les séries monétaires - tout l'intérêt qu'il porte aux provinces, et pas seulement les provinces de la sphère grecque même s'il y séjourne davantage et si son philhellénisme est grand. Les reliefs représentant les provinces qui ornaient son temple à Rome après sa mort rendent bien compte de cet intérêt majeur. Les monnaies du type Aduentus où il apparaît le plus souvent dans sa dimension civile de princeps, en toge, plutôt qu'en imperator célèbrent son arrivée dans de nombreuses contrées. D'autres, du type Restitutor, montrent l'empereur relevant une région de l'empire représentée par un personnage à genoux devant lui. En outre, à la fin du règne, dans les années 134-137, il réalise une série monétaire spécialement consacrée aux provinces. Les provinces sont représentées comme des unités culturelles avec leur spécificité, chacune devant contribuer pour sa part à l'œuvre commune. Chaque membre a son rôle à tenir dans l'organisation de l'ensemble, rôle qu'il appartient à l'empereur de lui assigner pour réaliser l'unité dans la diversité. Cette conception de l'État, dont le voyage impérial est un révélateur essentiel, coïncide avec l'universalisme de la pensée politique cynico-stoïcienne d'un Épictète.

Les voyages d'Hadrien ne sont donc pas le fait d'un dilettante, d'un esthète attentif à ses plaisirs, ils sont un moyen de gouverner avec efficacité pour un prince qui aime la paix et, par conséquent, sait préparer son armée à la guerre et assurer les défenses de l'empire (Vie d'Hadrien, 11.1-2), un prince qui sait que gouverner c'est aller voir sur place pour décider au mieux, que c'est se montrer pour renforcer l'unité de ce monde immense, un prince qui a compris que Rome n'est plus seulement dans Rome, mais qu'elle va du centre à la périphérie. Dion Cassius (69.9) met l'accent sur le zèle d'Hadrien à gouverner efficacement et à se donner en exemple lors de ses voyages.

Les sophistes proclament aussi, vers la même époque, l'unification du monde sous l'autorité de l'empereur ; c'est ainsi que, sous le règne du successeur d'Hadrien, Aelius Aristide, célébrant Rome, comparera l'oikouménè à une vaste cité : “Une démocratie commune à la terre est instaurée sous l'autorité unique du meilleur gouvernant et ordonnateur, et tous 
convergent ici, comme vers une commune agora, pour obtenir chacun ce qu'il mérite" (Rom., 6o, trad. Laurent Pernot, Les Belles Lettres, 1997). Mais Hadrien, au lieu de faire venir le monde à Rome, fait que Rome, qu'il incarne, va vers le monde.

\section{LE VOYAGEUR INFINI}

Le goût du voyage est, en fait, profondément ancré chez le personnage historique et c'est l'un des nombreux points de rencontre entre Marguerite Yourcenar et lui. Les grands personnages yourcenariens sont de grands voyageurs : Zénon, le héros de L'OEuvre au Noir, avant de s'arrêter définitivement à Bruges, parcourt le monde pendant très longtemps, en quête de savoir, disant : "Qui serait assez insensé pour mourir sans avoir fait au moins le tour de sa prison ?” $(O N, \text { p. 564 })^{29}$. Et Nathanaël, le protagoniste d'Un homme obscur, est entraîné malgré lui dans une sorte de tour du monde, avant de revenir à Amsterdam. La particularité d'Hadrien est d'être en même temps le maître du monde : "Quelques hommes avant moi avaient parcouru la terre : Pythagore, Platon, une douzaine de sages, et bon nombre d'aventuriers. Pour la première fois, le voyageur était en même temps le maître, pleinement libre de voir, de réformer, de créer. C'était ma chance, et je me rendais compte que des siècles peut-être passeraient avant que se reproduisît cet heureux accord d'une fonction, d'un tempérament, d'un monde" (Had., p. 382). Le voyage est ainsi, pour lui, également mise en ordre du monde : c'est une façon de contribuer à l'ordre olympien qu'il incarne contre les forces du chaos.

Mais, chez Marguerite Yourcenar, l'aspiration d'Hadrien au voyage va beaucoup plus loin, comme le montre la phrase qui suit immédiatement : "Et c'est alors que je m'aperçus de l'avantage qu'il y a à être un homme nouveau, et un homme seul, fort peu marié, sans enfants, presque sans ancêtres, Ulysse sans autre Ithaque qu'intérieure" (Had., p. 382) : le voyage est l'expression de la liberté. Arrien dans son Anabase d'Alexandre le Grand souligne la passion insatiable du Macédonien qui le pousse à vouloir découvrir ce qu'il y a au-delà, mais pour le conquérir, dans un immense désir de gloire (1.3.7.1 ; 7.16). L'Hadrien de Marguerite Yourcenar subit lui aussi la tentation du voyage infini, mais dans un sens totalement différent. Marguerite Yourcenar prête au jeune Hadrien, avant son accession, une tentation étrange, à la suite du don d'une pierre verte provenant d'un royaume inconnu : ce personnage, devenu pour nous une figure emblématique et un extraordinaire passeur de la civilisation grécoromaine, aurait fait le "rêve monstrueux" (Had., p. 323) de sortir du monde gréco-romain et de s'affranchir de tout lien culturel pour partir, dans un souci de liberté totale, à la découverte de l'ailleurs, du tout autre : "Je jouais avec cette idée... être seul, sans biens, sans prestiges, sans aucun des bénéfices d'une culture, s'exposer au milieu d'hommes neufs et parmi des hasards vierges... Il va de soi que ce n'était qu'un rêve, et le plus bref de tous. Cette liberté que j'inventais n'existait qu'à distance ; je me serais bien vite recréé tout ce à quoi j'aurais renoncé" (Had., p. 323). C'est donc à l'intérieur de l'empire qu'il voyagera. Mais on voit bien l'importance du jeu temporel occasionné par la distance entre le temps vécu d'Hadrien et le présent de l'écriture de son auteur, entre le $\mathrm{II}^{\mathrm{e}}$ et le $\mathrm{Xx}^{\mathrm{e}} \mathrm{s}$. de notre ère. L'espace de l'orbis 
terrarum antique semble trop étroit pour Hadrien et Marguerite Yourcenar lui fait pressentir d'autres horizons, qui sont les nôtres : "Nous connaissons encore assez mal la configuration de la terre. À cette ignorance, je ne comprends pas qu'on se résigne. J'envie ceux qui réussiront à faire le tour des deux cent cinquante mille stades grecs si bien calculés par Ératosthène, et dont le parcours nous ramènerait à notre point de départ" (Had., p. 323). Et, quand il se trouve en Bretagne, sortant par la pensée de son strict cadre chronologique grâce au recul de son auteur, il découvre en quelque sorte, à sa manière, l'Amérique :

"Chaque voyage déplaçait ainsi le centre de gravité du pouvoir, le mettait pour un temps au bord du Rhin ou sur la berge de la Tamise, me permettait d'évaluer ce qu'eussent été le fort et le faible d'un pareil siège impérial. Ce séjour en Bretagne me fit envisager l'hypothèse d'un état centré sur l'Occident, d'un monde atlantique. Ces vues de l'esprit sont démunies de valeur pratique : elles cessent pourtant d'être absurdes dès que le calculateur s'accorde pour ses supputations une assez grande quantité d'avenir". (Yourcenar, Had., p. 393)

Quand l'âge et la maladie lui interdisent les voyages, son goût de l'ailleurs ne l'abandonne pas pour autant : la Villa Adriana qu'il a fait bâtir à Tibur, une soixantaine d'hectares de constructions pour un ensemble de 120 hectares, le ramène à l'univers du voyage. Si l'on en croit la Vie d'Hadrien :

Tiburtinam uillam mire exaedificauit, ita ut in ea et prouinciarum et locorum celeberrima nomina inscriberet, uelut Lycium, Academian, Prytanium, Canopum, Poecilen, Tempe uocaret. Et, ut nihil praetermitteret, etiam inferos finxit.

"Il fit bâtir sa merveilleuse villa de Tibur, conçue de telle sorte que les noms des provinces et des lieux les plus célèbres s'y trouvassent attribués ; c'est ainsi qu'il donna, par exemple, les dénominations de Lycée, d'Académie, de Prytanée, de Canope, de Poecile, de Tempé ; et, pour ne rien laisser de côté, il représenta même les Enfers". (Vie d'Hadrien, 26.5)

Il ne s'agit pas de reproduire fidèlement les lieux célèbres, ni de concentrer des merveilles - fût-ce en modèle réduit - dans l'espace, somme toute, restreint de la centaine d'hectares de la Villa ; mais ces sites, ainsi dénommés, fonctionnent comme signes, comme symboles renvoyant au voyage. Dans la tradition de l'art des jardins, "Hadrien avait moins tenté une reproduction exacte de ses modèles qu'une véritable symbolisation" ${ }^{30}$. Mais c'est aussi une façon de s'approprier le monde par un voyage immobile. Si les voyages impériaux ont en quelque sorte construit symboliquement le monde, il se trouve condensé et mémorisé dans la Villa. Les travaux de Jean-Claude Grenier, par exemple, ont montré que le "Sérapeum" de la Villa constitue une évocation géographique et mentale de l'Égypte et le bassin du "Canope" une image de la Méditerranée ${ }^{31}$. Pour l'Hadrien yourcenarien, la Villa est en grande partie le lieu du souvenir :

"J'avais doté chacun de ces édifices de noms qui évoquaient la Grèce : le Poecile, l'Académie, le Prytanée. Je savais bien que cette petite vallée plantée d'oliviers n'était pas Tempé, mais j'arrivais à lâge où chaque beau lieu en rappelle un autre, plus beau, où chaque délice s'aggrave du souvenir de délices passées. J'acceptais de me livrer à cette nostalgie qui est la mélancolie du désir. J'avais même donné à un coin particulièrement sombre du parc le nom de Styx, à 
une prairie semée d'anémones celui de champs Élysées, me préparant ainsi à cet autre monde dont les tourments ressemblent à ceux du nôtre, mais dont les joies nébuleuses ne valent pas nos joies. (Yourcenar, Had., p. 482-483)

Mais, paradoxalement, c'est alors même qu'il est reclus à la Villa, qu'il effectue ses plus grands voyages : non seulement, il revit, grâce au souvenir, ses pérégrinations passées :

"Ma chambre secrète au centre d'un bassin de la Villa n'est pas un refuge assez intérieur : j'y traîne ce corps vieilli ; j'y souffre. Mon passé, certes, me propose çà et là des retraites où j'échappe au moins à une partie des misères présentes : la plaine de neige au bord du Danube, les jardins de Nicomédie, Claudiopolis jaunie par la récolte du safran en fleur, n'importe quelle rue d'Athènes, une oasis où des nénuphars ondoient sur la vase, le désert syrien à la lueur des étoiles au retour du camp d'Osroès".(Yourcenar, Had., p. 500-501)

Mais là n'est pas l'essentiel : dans cette ultime retraite de la Villa, à laquelle on a donné, à tort, le nom de "Théâtre maritime", l'esprit d'Hadrien voyage beaucoup plus loin en une échappée hors du temps, grâce au mythe ; en effet, il repense alors au texte que lui a adressé Arrien, qui lui parlait d'une île, où "l'ombre de Patrocle apparaît aux côtés d'Achille" (p. 500): Hadrien voit là "une image de [s]a vie telle qu['il] aurai[t] voulu qu'elle fût" ; "Vue par lui, l'aventure de mon existence prend un sens, s'organise comme dans un poème ; l'unique tendresse se dégage du remords, de l'impatience, des manies tristes comme d'autant de fumées, d'autant de poussières ; la douleur se décante ; le désespoir devient pur. Arrien m'ouvre le profond empyrée des héros et des amis" (Had., p. 500). Sa passion tragique pour Antinoüs se trouve ainsi sublimée. Il peut vraiment dire : "je ne suis pas toujours à Tibur quand j'y suis" (Had., p. 306) et "Arrien m'offre mieux. À Tibur, du sein d'un mois de mai brûlant, j'écoute sur les plages de l'île d'Achille la longue plainte des vagues ; j'aspire son air pur et froid ; j'erre sans effort sur le parvis du temple baigné d'humidité marine ; j'aperçois Patrocle... Ce lieu que je ne verrai jamais devient ma secrète résidence, mon suprême asile. J'y serai sans doute au moment de ma mort" (Had., p. 501).

Hadrien sera vraiment jusqu'au bout un voyageur, comme en témoignent ses ultima uerba chez Marguerite Yourcenar : "Tâchons d'entrer dans la mort les yeux ouverts..." (Had., p. 515). N'oublions pas que parfois sur les tombes antiques grecques figuraient des sandales, "comme un accessoire nécessaire pour le dernier voyage" ${ }^{32}$. 


\section{BIBLIOGRAPHIE}

André, J.-M. et M.-F. Baslez (1993) : Voyager dans l'Antiquité, Paris.

Baslez, M.-F. (2003) : "Voyager au-delà : la symbolique du voyage dans la pensée grecque”, in : Duchêne 2003. Beaujeu, J. (1955) : La religion romaine à l'apogée de l'Empire. I : La politique religieuse des Antonins, Paris. Bernand, É. (1960) : Les inscriptions grecques et latines du Colosse de Memnon, Paris.

Birley, A. R. (1997) : Hadrian the restless Emperor, Londres-New York.

- (2003) : "Hadrian's travels", in : de Blois et al. 2003, 425-441.

de Blois, L., P. Erdkamp, O. Hekster, G. de Kleijn et S. Mols, éd. (2003) : The representation and perception of Roman imperial power, Amsterdam.

le Bohec, Y., éd. (1994) : L’Afrique, la Gaule, la Religion à l'époque romaine. Mélanges à la mémoire de Marcel Le Glay, Bruxelles.

Börker, C. et R. Merkelbach (1980) : Die Inschriften von Ephesos, V, Bonn.

Bosworth, A. B. (1993) : “Arrian and Rome : the Minor Works", Aufstieg und Niedergang der römischen Welt, II, 34. 1, Berlin-New York, .

Charles-Gaffiot, J. et H. Lavagne, éd. (1999) : Hadrien. Trésors d'une villa impériale, Milan.

Chevallier, R. (1988) : Voyages et déplacements dans l'Empire romain, Paris.

Chevallier, R. et R. Poignault (1998) : L'empereur Hadrien, coll. “Que sais-je ?", Paris.

Drew-Bear, T. et F. Richard (1994) : “Hadrien et Érastos, nauclère d'Éphèse”, in : le Bohec 1994, 742-751.

Duchêne, H., éd. (2003) : Voyageurs et Antiquité classique, Dijon.

Grenier, J.-C. (1989) : “La décoration statuaire du ‘Sérapeum' du 'Canope’ de la Villa Adriana”, MEFRA, 101-102, 925-1019.

— (1999) : “Le 'Sérapeum’ et le ‘Canope' : une ‘Égypte’ monumentale et une 'Méditerranée”, in : CharlesGaffiot \& Lavagne 1999, 75-77.

Grimal, P. (1969) : Les Jardins romains, Paris.

Gutsfeld, A. (2000) : “Hadrian als Jäger. Jagd als Mittel kaiserlicher Selbstdarstellung”, in : Martini 2000, 79-99.

Halfmann, H. (1986) : Itinera principum. Geschichte und Typologie der Kaiserreisen im römischen Reich, Stuttgart.

Kurtz, D. C. et J. Boardman (1971) : Greek burial customs, Londres.

Le Bohec, Y., éd. (2003) : Les discours d'Hadrien à l'armée d'Afrique : exercitatio, Paris.

Martini, W., éd. (2000) : Die Jagd der Eliten in den Erinnerungskulturen von der Antike bis in die frühe Neuzeit, Göttingen.

Preisendanz, K. (1928) : Die Griechische Zauberpapyri, I, Leipzig.

Roman, Y. (2008) : Hadrien. L'empereur virtuose, Paris.

Smallwood, E. M. (1966) : Documents illustrating the principate of Nerva, Trajan and Hadrian, Cambridge. 
